\title{
TO INVESTIGATE AND ASSES WHY SOME FOOD/ DIETARY SUPPLEMENTS ARE HEALTH HAZARDOUS
}

\author{
Neha Rani, *Bhanu PS Sagar, Sandeep Kumar Shukla \\ Department of Pharmacy, IEC Group of Institutions, Greater Noida, India \\ *Corresponding Author's: Email address: bpssagar@gmail.com
}

Received 12 Dec 2012; Review Completed 13 Jan 2013; Accepted 13 Jan 2013, Available online 15 Jan 2013

\begin{abstract}
Toxic ingredients of Pharmaceutical as well as Food grade food supplements / Dietary Supplement are Pygeum, Caffeine / Caffeine extract, Omega 3 fatty acid (source fish oil, flaxseed oil, or cod liver oil), Ephedrine (Ephedra sinica), Usnic acid, Kava-Kava (Piper methysticum), Gugulipid (Comiphora mukul), Liquorice (Glycyrrhiza glabra), Ginseng (Panax ginseng, Panax japonicus, Panax trifolius, Panax zingiberensis, Panax bipinnatifidus), St. John's wort (Hypericum perforatum) etc. Caffeine (1,3,7-trimethylxanthine) is an alkaloid of the methylxanthine family known as a central nervous system stimulant through its adenosine antagonist action. Caffeine is a naturally found in the leaves, seeds and/or fruits of at least 63 plant species worldwide. Caffeine produces psychoactive responses (alertness, mood change), neurological condition (infant hyperactivity, Parkinson's disease), metabolic disorders (diabetes, gallstones), and gonad and liver function. However, high doses may produce negative effects in some sensitive individuals, including anxiety, tachycardia and insomnia recommended upper limits of caffeine for healthy adults below 300-500 mg daily, pregnant women must stay below 150-200 mg daily and children should stay below $50 \mathrm{mg}$ daily. Amounts exceeding 700 milligrams of caffeine can be dangerous.
\end{abstract}

Key words: Caffeine, CNS stimulant

\section{INTRODUCTION}

A dietary supplement, also known as food supplement or nutritional supplement, is a preparation intended to supplement the diet and provide nutrients, such as vitamins, minerals, fatty acids, or amino acids that may be missing or may not be consumed in sufficient quantities in a person's diet. Some countries define dietary supplements as foods, while in others they are defined as drugs or natural health products. Food supplements make up nature's richest sources of natural vitamins, minerals and nutrients. The extensive range of food supplements are available as tablets, capsules, soft gels and liquid extracts food supplements are one of the best selling products on the market these days e.g. vitamins, minerals, herbs and amino acids etc.

A growing concern among physicians and consumers is whether dietary supplements contain all of the active ingredients listed on the label. This apprehension is based on well-publicized analyses of commercial supplements showing considerable variation in ingredient quality and quantity.

A deceptive practice that occurs in the ingredient industry is to submit samples that meet pharmaceutical standards, but not deliver the same high quality material for use in the finished dietary supplement.

Pharmaceutical grade refers to processes which ensure that a substance contains the amount of active ingredient specified and does not contain adulterants, toxins, heavy metals or contaminants. It costs more to produce supplements (or drugs) that are pharmaceutical grade as compared to lesser grades such as food grade, and not all supplements are pharmaceutical grade. Pharmaceutical grade supplements are more expensive reflecting their higher purity and quality. Our ingredients are purchased solely for quality and efficacy. They are sourced only from reputable trusted industry leaders.

The term pharmaceutical grade was originally taken from the standard of certification of the facilities in which pharmaceutical grade supplements were made. Pharma Nutrients' supplements are made in facilities registered with the FDA, by companies certified to produce prescription drugs. Pharma Nutrients full range of Omega-3 products are manufactured under "Good Manufacturing Practice" or GMP regulations which is part of a quality system covering the manufacture and testing of active pharmaceutical ingredients, diagnostics, foods, pharmaceutical products, and medical devices. GMPs are guidelines that outline the aspects of production and testing that can impact the quality of a product e.g. cod liver oil, fish oil, caffeine, soy milk etc. Some Hazards of Pharmaceutical Grade Food Supplements are

\section{MATERIALS AND METHODS}

\section{Selection and Procurement of Raw Materials.}

Following raw materials were procured from different commercial sources:

\begin{tabular}{|c|l|l|}
\hline S. No. & \multicolumn{1}{|c|}{ Name of Product } & \multicolumn{1}{c|}{ Name of Company } \\
\hline 1. & Tea Gallery (Fig. 5.1) & Pamah Exports Pvt. Ltd., Delhi \\
\hline 2. & Green Tea (Chundavurrai) (Fig. 5.2) & Kanan Devan Hills Plantations Company Pvt. Ltd. \\
\hline 3. & Energy (Assam Green Tea) (Fig. 5.3) & A. N. Securities, Pvt. Ltd., Kolkatta \\
\hline 4. & GAIA Tea (Fig. 5.4) & Cosmic Nutracos Solution Pvt. Ltd., Delhi \\
\hline
\end{tabular}




\begin{tabular}{|c|l|l|}
\hline 5. & Benefit Slimming Tea (Fig. 5.5) & Kunming Kanaking Trading Co. Ltd., China \\
\hline 6. & Easy Slim Tea (Fig. 5.6) & Easytvshop Trading Company \\
\hline 7. & Yogi Green Tea ((Fig. 5.7) & 950, International Way, Springfield \\
\hline 8. & Yogi Green Tea Decaf Kombucha (Fig. 5.8) & 950, International Way, Springfield \\
\hline
\end{tabular}

\section{Extraction Isolation and Purification of Caffeine from Herbal Teas used as Dietary Supplements.}

\section{Scheme for Caffeine Extraction}

The coarsely powdered tea leaves / dust (10 g) samples were separately extracted with boiling water and the aqueous extracts were filtered while hot.

The hot extracts were treated with $10 \%$ lead acetate solution to precipitate tannins as lead tannate. The excess of lead acetate present in the extracts were removed by precipitation with sulphuric acid as lead sulphate.

The filtered solutions were boiled with charcoal to remove the coloured impurities and filtered to remove charcoal.

The filtered decolourised solutions were extracted (solvent-solvent extraction / partitioning) with chloroform in separating funnels.

The chloroform extracts were evaporated to obtain caffeine as a white crystalline powder. Isolated caffeine was then recrystallised with alcohol. Murray, Hansen., et al

\section{Chemical Tests}

(a) Isolated caffeine samples were subjected to murexide colour reaction. Isolated caffeine samples were taken separately in petridishes to which hydrochloric acid and potassium chlorate were added and heated to dryness. Residues of the petridishes were exposed to vapours of dilute ammonia. Purplish colouration produced indicated the presence of caffeine. Further, on addition of fixed alkali the purple colouration disappeared.

(b) Isolated caffeine when treated with tannic acid solution produced white precipitate. Barry et al.

(ii) Melting Point determination of isolated Caffeine.

Melting point / mixed point were determined for isolated caffeine of various samples.

\section{Observations}

Presence / Absence of caffeine in different tea samples.

\begin{tabular}{|c|l|c|}
\hline S. No. & \multicolumn{1}{|c|}{ Name of Product } & Caffeine Present / Absent \\
\hline 1. & Tea Gallery & - \\
\hline 2. & Green Tea (Chundavurrai) & + \\
\hline 3. & Energy (Assam Green Tea) & + \\
\hline 4. & GAIA Tea & + \\
\hline 5. & Benefit Slimming Tea & + \\
\hline 6. & Easy Slim Tea & + \\
\hline 7. & Yogi Green Tea & - \\
\hline 8. & Yogi Green Tea Decaf Kombucha \\
\hline
\end{tabular}

\section{Thin Layer Chromatographic analysis of Caffeine}

The thin layer chromatography (TLC) technique was used to detect the presence of caffeine. Samples were prepared by dissolving $1 \mathrm{mg}$ of caffeine in $1 \mathrm{ml}$ of chloroform or methanol. The solvent system / mobile phase used were ethyl acetatemethanol-acetic acid (80:10:10). To visualise the spots of caffeine dried TLC plates were exposed to iodine vapour.

\section{Ultraviolet (UV) Spectroscopic analysis of isolated Caffeine.}

For UV analysis the caffeine samples were dissolved in methanol and UV absorption / spectroscopic analysis was carried out to find out the $\lambda_{\max }$. Systronics (model 2202) Double beam instrument was used for UV analysis of caffein

\section{Observations}

In TLC analysis, mobile phase comprising of Ethyl acetate-methanol-acetic acid (80:10:10) was found suitable for the separation of caffeine. Brown colour spots were observed when Dried TLC plates were exposed to iodine vapour and caffeine developed spot at $R_{f}$ value 0.41. Mobile phase with Ethyl acetate-methanol (80:20) was also found suitable for separation of caffeine with $\mathrm{R}_{\mathrm{f}}$ value 0.46

Spectrophotometry provides a sensitive method for the detection and measurement of caffeine. The UV absorption spectrum of caffeine exhibits a pair of absorption bands peaking at $205 \mathrm{~nm}$ and $273 \mathrm{~nm}$ with a characteristic absorption shoulder between them (Fig. 5.10-5.11). The $\lambda \max$ was found to be $275 \mathrm{~nm}$ and the absorption was $100 \%$. Gillyon., et al. 


\section{Instruments used}

\section{Shimadzu FTIR 8400S}

IR spectra were recorded on a Shimadzu FTIR 8400S in KBr pellets in the Central Instrumentation Facility (CIF) lab of the department. FTIR-8400S is combined with the IRsolution - a 32 bit high performance FTIR software - to analyze your samples easily and securely.

\section{NMR Spectroscopic Instruments}

For NMR (proton and carbon) and Mass spectral analysis of purified caffeine samples were sent to IIT, Delhi and Jubilant Chemesys Pvt. Ltd., Noida. The High Resolution NMR spectrometer (Bruker, $300 \mathrm{MHz}$ ) was used to record the various type of $1 \mathrm{D}\left({ }^{1} \mathrm{H},{ }^{13} \mathrm{C}\left\{{ }^{1} \mathrm{H}\right\}\right.$, DEPT) NMR spectra for structure elucidation of isolated caffeine.

\section{IR spectroscopy}

Potassium bromide $(\mathrm{KBr})$ pellets of caffeine was prepared and used for FTIR analysis using Shimadzu FTIR 8400S at Central Instrumentation Facility (CIF) lab of the department.

\section{NMR Spectroscopy}

Caffeine was dissolved in $\mathrm{CDCl}_{3}$ and subjected to NMR (proton and carbon) analysis using High Resolution NMR spectrometer (Bruker, 300MHz) at IIT, Delhi and Jubilant Chemesys Pvt. Ltd., Noida. Finally, the spectral elucidation was carried by expert organic chemists / professors. ${ }^{1} \mathrm{H}$ and ${ }^{13} \mathrm{C}$ NMR spectra were acquired using Bruker Advance 400 and 500 NMR spectrometer

Proton and Carbon spectral data of purified caffeine.

\section{Observations}

\begin{tabular}{|c|l|c|}
\hline Position & Caffeine $\boldsymbol{\delta}_{\mathbf{C}}(\mathbf{J}$ in Hz) & Caffeine $\boldsymbol{\delta}_{\mathbf{H}}$ \\
\hline 1 & $\mathrm{~N}$ & - \\
\hline 2 & 151.45 & - \\
\hline 3 & $\mathrm{~N}$ & - \\
\hline 4 & 148.46 & - \\
\hline 5 & 107.31 & - \\
\hline 6 & 155.13 & - \\
\hline 7 & $\mathrm{~N}$ & - \\
\hline 8 & - & 7.26 \\
\hline $1^{\prime}$ & $\mathrm{NCH}_{3}-29.5$ & 3.591 \\
\hline $3^{\prime}$ & $\mathrm{NCH}_{3}-27.6$ & 3.414 \\
\hline $7^{\prime}$ & $\mathrm{NCH}_{3}-33.36$ & 4.232 \\
\hline
\end{tabular}

IR (KBr): IR spectra were recorded on a Shimadzu FTIR 8400S in KBr pellets. FT-IR spectrometer

(Spectrum 1000), and absorbance frequencies are reported in reciprocal centimeters ( $\left.\mathrm{cm}^{-1} 1\right)$. The spectra showed following functional groups / bond peaks: C-C Str. 980, CH bonding 1400, C-N Str. 1550, C-O 1700, CH Str. 2960 , NH-Str. 3120.

MS : Caffeine: m.wt. 195

NMR $\left({ }^{1} \mathrm{HNMR}\right.$ and $\left.{ }^{13} \mathrm{CNMR}\right)$ (DMSO-d6) data:

${ }^{1} \mathrm{HNMR}\left(\mathrm{CDCl}_{3}\right)$ data showed following peaks / values: C-8 (7.26), C-1' $\left(\mathrm{NCH}_{3}-3.591\right), \mathrm{C}-3$ '

$\left(\mathrm{NCH}_{3}-3.414\right), \mathrm{C}-7$ ' $\left(\mathrm{NCH}_{3}-4.232\right)$.

${ }^{13} \mathrm{CNMR}\left(\mathrm{CDCl}_{3}\right)$ data showed following peaks / values: C-1 (N), C-2 (151.45), C-3 (N), C-4 (148.46), C-5 (107.31), C-6 (155.13), C-7 (N), C-8 (-), C-1' ( $\left.\mathrm{NCH}_{3}-29.5\right), \mathrm{C}-3^{\prime}\left(\mathrm{NCH}_{3}-27.6\right), \mathrm{C}-7^{\prime}\left(\mathrm{NCH}_{3}-33.36\right)$.

Spectral elucidation of the isolated caffeine samples confirms its presence of caffeine in samples number 2-7.

Ultra High Performance Liquid Chromatography - Mass spectrometric (UHPLC-MS) analysis for detection and quantitative estimation of Caffeine from Herbal Teas.

Instrument used for Ultra High Performance Liquid Chromatography - Mass spectrometric (UHPLC-MS)

A Waters UHPLC system of Alliance 2695 (Milford, MA, USA) was equipped with quaternary gradient pumps, auto-sampler and a photo diode array detector (PDA 2996). This HPLC system was connected to Waters QToF micro mass spectrometer (Milford, MA, USA) and operated with MassLynx software. Samples were analyzed with blinded standard reference materials for caffeine content by ultra high performance liquid chromatography with Mass analysis. 
For mass spectrometry detection, the parameters were tuned by injecting low concentrated caffeine standard for better mass sensitivity. QToF mass spectrometer was provided with electrospray ionization source and operating parameters were as follows: Capillary voltage, $3500 \mathrm{~V}$; sample cone voltage, $35 \mathrm{~V}$; extraction cone voltage, $2.0 \mathrm{~V}$. Collision energy was initially set at $5.0 \mathrm{eV}$ for MS experiments and varied from 5 to $30 \mathrm{eV}$ for MS/MS experiments. Nitrogen gas was used as nebulizer gas and Helium gas as collision gas. Nebulizer gas was supplied with a flow rate of $50 \mathrm{~L} / \mathrm{hr}$ and dry gas of 500 $\mathrm{L} / \mathrm{hr}$. In the time-of-flight analyzer, the flight tube voltage was set at $5630 \mathrm{~V}$ and the detector voltage was $2550 \mathrm{~V}$. All the experiments were carried out in the positive ionization mode.

\section{Procedure}

Isolated and purified caffeine was subjected to ultra high performance liquid chromatographic with mass spectrometer (UHPLC-MS) analysis for its identification and quantitative estimations. The UHPLC-MS analyses were carried out in Jubilant Chemesys Pvt. Ltd., Noida. The analysis was performed on non-polar C18 column (250 x $4.6 \mathrm{~mm})$ utilizing a gradient elution profile. The eluent was acetonitrile-water gradient at a flow rate of $1.0 \mathrm{ml} / \mathrm{min}$ and UV detection at 275 $\mathrm{nm}$. This method was used in the quantification of caffeine in various samples of Herbal teas. Caffeine was identified by comparing the retention times and spectral data with those of authentic standards.

\section{Preparation of Standard Curve}

A series of caffeine dilutions were prepared like $2 \mathrm{mg} / \mathrm{ml}, 4 \mathrm{mg} / \mathrm{ml}, 6 \mathrm{mg} / \mathrm{ml}, 8 \mathrm{mg} / \mathrm{ml}$, and $10 \mathrm{mg} / \mathrm{ml}$. Standards that bracket the unknown sample concentration were measured to construct a calibration curve. A comparison of the caffeine peak area in the herbal teas sample compared to those for the standards permits a quantitative determination of the caffeine content.

Standard Plot of Caffeine using UHPLC-MS with retention time, concentration, area and Molecular mass.

\begin{tabular}{|c|c|c|c|c|c|}
\hline S. No. & Retention Time (min.) & $\begin{array}{c}\text { Concentration } \\
(\mathbf{m g} / \mathbf{m l})\end{array}$ & Area & Mass & Fig. no. \\
\hline 1 & 0.924 & 2 & 1011.414 & 195.0 & $5.17-5.18$ \\
\hline 2 & 0.924 & 4 & 1791.032 & 195.0 & $5.19-5.20$ \\
\hline 3 & 0.924 & 6 & 2352.822 & 195.0 & $5.21-5.22$ \\
\hline 4 & 0.924 & 8 & 3063.161 & 195.0 & 5.23 \\
\hline 5 & 0.924 & 10 & 3714.986 & 195.0 & 5.24 \\
\hline
\end{tabular}

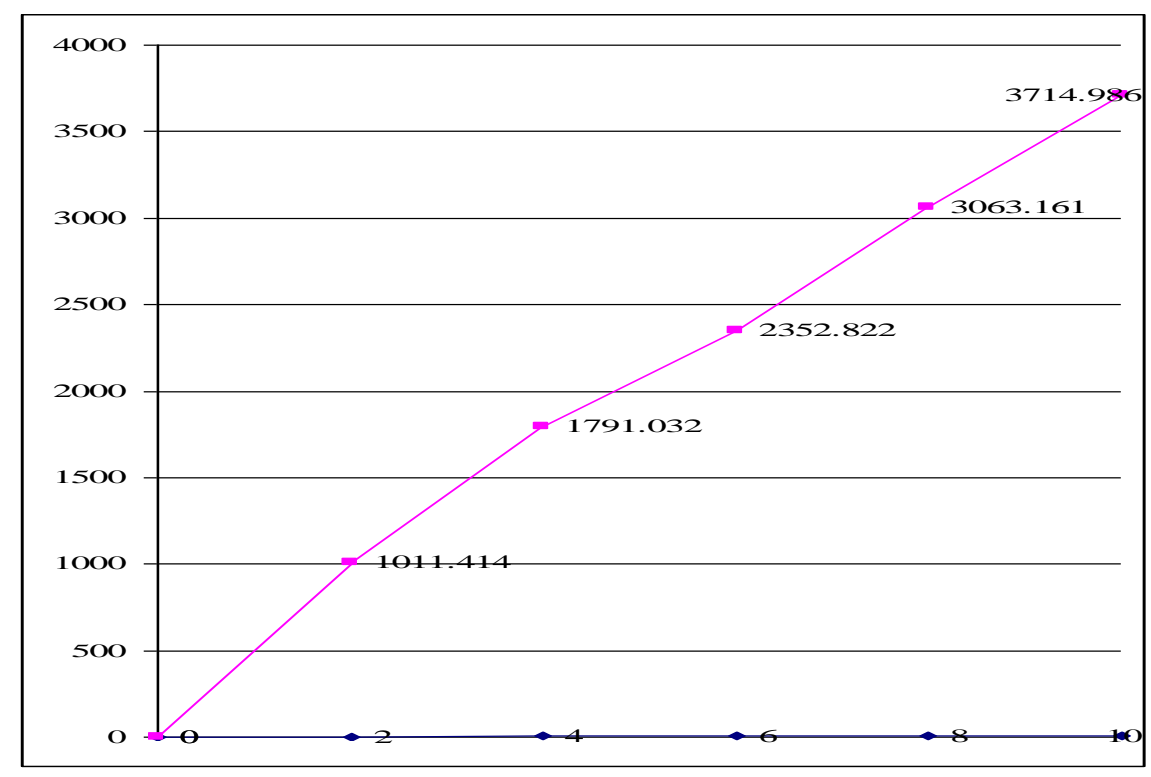

Fig: Standard Plot of caffeine using UHPLC-MS.

\section{Sample Preparation}

Tea samples $(2.5 \mathrm{~g})$ were poured with $20 \mathrm{ml}$ of boiling water and stirred for 10 minutes. Extracts were filtered through a cotton wool, cooled at a room temperature, diluted with $25 \mathrm{ml}$ distilled water and used for UHPLC-MS analysis.

\section{Observations}

The chromatographic and spectral analyses confirmed that the isolated and purified compound was caffeine. Sharp peaks with good resolution were attained using $1.0 \mathrm{ml} / \mathrm{min}$. The optimized injection volume and detection wavelength were $10 \mu \mathrm{l}$ and $275 \mathrm{~nm}$, respectively.

\section{Mass accuracy}


The difference between theoretical and experimental masses is generally expressed in terms of mass accuracy. The accurate mass measurement gives the elemental composition of the parent and fragment ions and was used to identify unknown species. QToF-MS permits MS/MS analysis and provides accurate masses for both precursor and product ions, which constitutes a higher order mass identification than those afforded by nominal mass measurements obtained by other types of mass analyzers. In QToF, the selectivity of precursor ion scans is very high because the high resolving power of the reflectron-ToF mass analyzers provides high accuracy fragment ions without compromising sensitivity

The elemental composition was a complement from the interpreted fragmentation pattern and the accurate mass measurement of the obtained product ions, thus relieving existing equivocalnesses. Mass measurement accuracy along with characteristic retention time, usually provides highly reliable identification of target species

In UHPLC-MS analysis, separation was achieved in 0.924 min with a water-acetonitile. Detection was effected with UV detector at $275 \mathrm{~nm}$.

\section{CONCLUSION:}

Different Dietary tea supplements were selected and tested for the presence / concentration of the toxic constituents like caffeine. Both qualitative and quantitative assessment of toxic constituents were carried out by making use of advanced scientific methods using instruments like Double Beam UV, HPLC, FTIR, Mass and NMR. The caffeine content of the tested herbal teas determined by UHPLC-MS analysis was decreasing in the following order: Energy (Assam Green Tea) $(3.62 \%)>$ Green Tea (Chundavurrai) $(3.18 \%)>$ GAIA Tea $(2.79 \%)>$ Benefit Slimming Tea $(2.77 \%)>$ Yogi Green Tea $(2.35 \%)>$ Easy Slim Tea $(1.13 \%)$. Conclusively, the caffeine content of dietary supplements is not always listed on the label, these products can provide as much or more caffeine (toxic concentrations) than a cup of brewed coffee / tea.

\section{ACKNOWLEDGEMENT:}

Author wants to thanks IEC Group of Institution for their infrastructural support in this study. I also express my gratitude to all the faculty members of department of Pharmacognosy, Manipal University for their guidance in writing this manuscript.

\section{REFERENCES}

1. Abernethy DR and Todd EL., 1985. Impairment of caffeine clearance by chronic use of low-dose oestrogen-containing oral contraceptives. Eur J Clin Pharmacol., vol 28.

2. Akhtar S, Wood G and Rubin JS., 1999. Effect of caffeine on the vocal folds: a pilot study. J Laryngol Otol., vol 113.

3. Ali M and Afzal M., 1987. A potent inhibitor of thrombin stimulated platelet thromboxane formation from unprocessed tea. Prostaglandins Leukot Med., vol 27.

4. Anderson BJ, Gunn TR, Holford NH and Johnson R., 1999 Caffeine overdose in a premature infant: clinical course and pharmacokinetics. Anaesth Intensive Care., vol 27.

5. Dr. N. Singh, Bhavani Lev, Bharat Mitra, Indrajeet Singh and Kailash Nath Singh., 1997. Website: http://www.Organic india.com/organic supplements.

6. Larimore WL and O'Mathúna DP., 2003. "Quality assessment programs for dietary supplements". The Annals of Pharmacotherapy., vol 37 (6).

7. Veierod MB, Thelle DS and Laake P., 1997. Diet and risk of cutaneous malignant melanoma: a prospective stu dy of 50,757 Norwegian men and women. Int $J$ Cancer., vol 71.

8. Zeratsky K., 2011. Mayo Clinic, Rochester, Minn, weblivsite: http://www.livestrong.com/ Supplements Vs Whole foods.

9. Murray, D.S.; Hansen, P.J., 1995. J. Chem. Educ., vol 72 (851)

10. Conte, E. D.; Barry, E. F.; Rubinstein, H. J. 1996. Chem. Educ., vol 73 (1169-1170)

11. Gillyon, E. C. P. Chromatogr. 1980. Newslett. Vol. 8 (50-51). 